\title{
Onsager approach to the one-dimensional solidification problem and its relation to the phase-field description
}

\author{
Efim A. Brener and D. E. Temkin \\ Peter Grünberg Institut, Forschungszentrum Jülich, D-52425 Jülich, Germany
}

(Received 13 December 2011; published 19 March 2012)

\begin{abstract}
We give a general phenomenological description of the steady-state 1D front propagation problem in two cases: the solidification of a pure material and the isothermal solidification of two-component dilute alloys. The solidification of a pure material is controlled by the heat transport in the bulk and the interface kinetics. The isothermal solidification of two-component alloys is controlled by the diffusion in the bulk and the interface kinetics. We find that the condition of positive-definiteness of the symmetric Onsager matrix of interface kinetic coefficients still allows an arbitrary sign of the slope of the velocity-concentration line near the solidus in the alloy problem or of the velocity-temperature line in the case of solidification of a pure material. This result offers a very simple and elegant way to describe the interesting phenomenon of a possible non-single-value behavior of velocity versus concentration that has previously been discussed by different approaches. We also discuss the relation of this Onsager approach to the thin-interface limit of the phase-field description.
\end{abstract}

DOI: $10.1103 /$ PhysRevE.85.031601

PACS number(s): 81.10.Aj, 64.70.dm, 81.30.Fb, 05.70.Ln

\section{INTRODUCTION}

In recent years the phase-field approach to solidification problems has attracted the attention of much research (see, for example, Ref. [1] and references therein). It was originally introduced as a mathematical tool to solve the free boundary problem without directly tracking the interface position. However, more recently it has also been considered as a physical model that can bring additional information compared to the sharp interface approach. In particular, it was observed that steady-state $1 \mathrm{D}$ front propagation with positive velocity,

$$
V=V_{0}\left(\Delta_{T}-1\right),
$$

is possible only if $\left(\Delta_{T}-1\right)>0$ (see, for example, Ref. [2]) is not the general situation. Here $V$ is the steady-state front velocity; $V_{0}$ is the characteristic velocity that is proportional to the kinetic growth coefficient; $\Delta_{T}=\left(T_{M}-T_{0}\right) c_{p} / L$ is the dimensionless undercooling; $T_{M}$ is the melting temperature; $T_{0}$ is the temperature in the original phase far from the interface; $c_{p}$ is the heat capacity, which is assumed to be the same in both phases; and $L$ is the latent heat. Karma and Rappel (KR) [3] introduced the thin-interface limit of the phase-field description and found that

$$
V=\frac{V_{0}\left(\Delta_{T}-1\right)}{1-a \frac{W V_{0}}{D_{T}}},
$$

where $D_{T}$ is the thermodiffusion coefficient, $a$ is a positive number of order unity that depends on the details of the model, and $W$ is the thickness of the interface in the phase-field description. In the phased field model discussed in Ref. [3], there is no any restriction on the parameter $V_{0} W / D_{T}$ and the velocity may be positive for $\left(\Delta_{T}-1\right)<0$. The same result was obtained for the isothermal solidification of alloys by many authors starting from a paper by Löwen et al. [4] in the framework of phase-field description and also by Aziz and Boettinger [5], who use a more phenomenological approach. In the case of alloys the deviation from equilibrium is defined by $\Delta_{C}=\left(C_{L}-C_{0}\right) /\left(C_{L}-C_{S}\right)$ instead of $\Delta_{T}$. In the two-phase region of the phase diagram, $0<\Delta_{C}<1$. Here $C_{L}$ and $C_{S}$ are the equilibrium concentrations of the initial and growing phase, respectively, and $C_{0}$ is the concentration of the initial phase far from the interface. They found that the steady-state growth is possible also inside the two-phase region of the equilibrium phase diagram.

From the numerous papers on the derivation of the sharp and thin-interface limits from a phase-field model we should also mentioned the work by Elder et al. [6] and Umantsev [7] and the paper by Korzhenevskii, Bausch, and Schmitz [8], which contains many details and technical points. The basic results of all these descriptions have the structure of Eq. (2) in the vicinity of $\left(\Delta_{T(C)}-1\right) \ll 1$ and eventually lead to the non-single-value behavior of the velocity as a function of the driving force in the case of a negative "kinetic coefficient" (Fig. 1). In this case the branch which is described by Eq. (2) (dotted line in Fig. 1) is linearly unstable (see, for example, Refs. $[3,4,8]$ ) while the "high velocity" branch of the mentioned non-single-value behavior is linearly stable.

Qualitatively the same results have been obtained by the numerical solution of $1 \mathrm{D}$ motion of the atomically rough interface in binary alloys [9]. In this model instead of the phase-field order parameter the authors used the fraction of the atomic places which belongs to the growing phase. This fraction changes from 0 to 1 during the growth. The evolution equations for this quantity together with the concentration fields in the two phases are given by Eqs. (5.1)-(5.3) in Ref. [10]. The numerical analysis of Ref. [9] has shown that both types of curves in Fig. 1 are possible. However, the unstable (dotted line) branch was not seen in this dynamical simulation.

An interesting explanation of a non-single-value behavior of velocity versus concentration suggested by the phase-field modeling and relative approaches cited above explicitly takes inhomogeneities of the concentration field, on the scale of finite interface thickness, into account. The purpose of this paper is to give a complementary phenomenological description of the steady-state 1D front propagation problem in two cases: (i) the solidification of a pure material, which is controlled by the heat transport in the bulk and the interface kinetics, and 


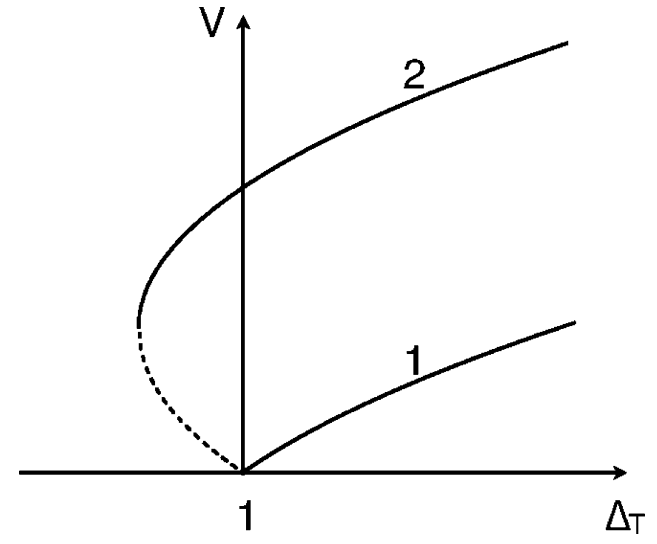

FIG. 1. Schematic dependence of the steady-state velocity $V$ vs. the dimensionless undercooling $\Delta_{T}$. Curve 1 corresponds to the case $a W V_{0} / D_{T}<1$ while curve 2 corresponds to the opposite case, $a W V_{0} / D_{T}>1$.

(ii) the isothermal solidification of two-component dilute alloys, which is controlled by the diffusion in the bulk and the interface kinetics. Describing the interface boundary conditions we use only the general phenomenology of linear nonequilibrium thermodynamics in the spirit of the Onsager matrix of kinetic coefficients that has the proper symmetry and is positive definite as required by the second law of thermodynamics. This approach does not assume any specific model of the interface and makes no assumption on its thickness. The only requirement, as for any macroscopic theory, is that the thickness is small compared the macroscopic lengths. We will see that two mentioned problems are very close to each other and can be formally mapped onto each other. The mentioned restrictions on the Onsager matrix of kinetic coefficients are not sufficient to determine the sign of the slope of the velocity-concentration line near the solidus in the alloy problem (or of the velocity-temperature line in the case of solidification of a pure material). This result offers a simple way to describe the mentioned above phenomenon of a non-single-value behavior (Fig. 1).

The sharp $(W \rightarrow 0)$ and the thin-interface limits of the phase-field description should lead to the effective macroscopic description with the boundary conditions in the spirit of Onsager relations, where the elements of the Onsager matrix are expressed in terms of the phase-field model parameters. Indeed, these limits really correspond to such a description. However, the mentioned condition of positive-definiteness of the matrix of kinetic coefficients turns out to be a nontrivial issue for the thin-interface limit and will be discussed in more detail.

\section{GROWTH OF A PURE MATERIAL WITH HEAT TRANSPORT}

We assume that phase 1 grows at the expense of phase 2 by a 1D front propagation with the steady-state velocity $V$. In the bulk we have the thermal-conductivity equation. In order to write down the boundary conditions at the interface, we follow the description and notations given in Ref. [11] as follows:

$$
\begin{gathered}
\left(\mu_{2}-\mu_{1}\right) / T_{M}=\mathcal{A} V+\mathcal{B} J_{E}, \\
\left(T_{2}-T_{1}\right) / T_{M}^{2}=\mathcal{B} V+\mathcal{C} J_{E},
\end{gathered}
$$

where $\mu_{i}$ is the chemical potential of the corresponding phase $i$ at the interface. According to the energy conservation at the interface (see also Eqs. (51) and (52) in Ref. [11]),

$$
\begin{aligned}
& -\lambda_{1} \nabla T_{1}=V T_{M} S_{1}-J_{E}, \\
& -\lambda_{2} \nabla T_{2}=V T_{M} S_{2}-J_{E} .
\end{aligned}
$$

Here $S_{1}\left(T_{1}\right)$ and $S_{2}\left(T_{2}\right)$ are the entropies of two phases and $\lambda_{i}$ is the thermoconductivity of phase $i$. The total heat flux $J_{E}$, flowing through the interface from phase 2 into phase 1 , consists of the following two parts: the heat flux due to the gradients of temperature and the energy flux due to the finite velocity of the interface that takes into account the different values of the entropy in each phase. We note that each contribution to the total flux is discontinuous at the interface while the total flux $J_{E}$ is, of course, continuous. This total flux flowing through the interface together with the growth velocity should be inserted in the linear relations between driving forces and fluxes, Eqs. (3) and (4).

The elements of the Onsager matrix, which is symmetric and positive definite, obey the conditions $\mathcal{A}, \mathcal{C}>0$ and $\mathcal{B}^{2}<$ $\mathcal{A C} . R_{K}=\mathcal{C} T_{M}^{2}$ is the Kapitza resistance and the cross coefficient $\mathcal{B}$ describes the way the two entropies are shared between the two sides of the interface during growth (for a more detailed discussion of the physical meaning of the different Onsager coefficients in this case, see Ref. [11]).

For the steady-state one-dimensional problem $\nabla T_{1}=0$ and $T_{1}=T_{0}+L / c_{p}$, where $L=T_{M}\left[S_{2}\left(T_{M}\right)-S_{1}\left(T_{M}\right)\right]$ is the latent heat and $c_{p}$ is the heat capacity, $T_{0}$ is the temperature in the original phase far from the interface. We note that in order to obtain the relation $T_{1}=T_{0}+L / c_{p}$ one should expand the entropies near the equilibrium temperature $T_{M}$ in the energy conservation condition $\left(\lambda_{1} \nabla T_{1}-\lambda_{2} \nabla T_{2}\right)=$ $V T_{M}\left[S_{2}\left(T_{2}\right)-S_{1}\left(T_{1}\right)\right]$. Now expanding the difference of the chemical potentials near the equilibrium temperature $T_{M}$, we find

$$
\mu_{2}\left(T_{2}\right)-\mu_{1}\left(T_{1}\right)=S_{2}\left(T_{M}\right)\left(T_{M}-T_{2}\right)-S_{1}\left(T_{M}\right)\left(T_{M}-T_{1}\right),
$$

and, finally, we get

$$
V=\frac{L^{2}\left(\Delta_{T}-1\right)}{c_{p} T_{M}^{2}\left[\mathcal{A}+\mathcal{C} T_{M}^{2} S_{1} S_{2}+\mathcal{B} T_{M}\left(S_{1}+S_{2}\right)\right]},
$$

where $\Delta_{T}=\left(T_{M}-T_{0}\right) c_{p} / L$. We have used the usual notation for solidification of pure materials. We see that the sign of $\left(\Delta_{T}-1\right)$ in general is not determined by the Onsager restriction $\mathcal{B}^{2}<\mathcal{A C}$. However, it is well defined in two cases: (i) $\mathcal{B}=0$ and (ii) in the "isothermal" case, $T_{1}=T_{2}$. In the later case the growth rate is controlled by the "isothermal" kinetic coefficient which is strictly positive due to the mentioned restriction, $\mathcal{B}^{2}<\mathcal{A C}[11]$ :

$$
V=\frac{\left(\mu_{2}-\mu_{1}\right)}{T_{M} \mathcal{A}\left[\left(1-\mathcal{B}^{2} /(\mathcal{A C})\right]\right.}=\frac{L^{2}\left(\Delta_{T}-1\right)}{c_{p} T_{M}^{2} \mathcal{A}\left[\left(1-\mathcal{B}^{2} /(\mathcal{A C})\right]\right.},
$$


Karma and Rappel obtained in their thin-interface limit $\mathcal{B}=\mathcal{C}=0$ and a coefficient $\mathcal{A}$ which may even be negative $(\beta$ in their notation). They discussed this "counterintuitive" issue and gave some natural explanation for this phenomenon. We will return to this point later.

\section{ISOTHERMAL ALLOY SOLIDIFICATION IN THE DILUTE LIMIT}

We discuss the steady-state propagation of a 1D front with velocity $V$ during solidification of a two-component alloy at a given temperature $T$. The concentration of $B$ atoms is $C_{1}(x)$ in phase 1 and $C_{2}(x)$ in phase 2 . In the bulk these concentrations are described by diffusion equations with diffusion coefficients $D_{1}$ and $D_{2}$. In order to write down the boundary conditions in this case we use the same phenomenological approach but adapted to the alloy situation. Onsager relations connect two fluxes $J_{A}$ and $J_{B}$ (at the boundary) of atoms $A$ and $B$ to two driving forces $\delta \mu_{A}$ and $\delta \mu_{B}$, which are the usual differences in chemical potentials at the boundary. While the bulk is described by diffusional equations for the concentration fields for each phase, we still need three boundary conditions at the interface. One is the conservation of $B$ atoms at the interface. We have also to relate the two concentrations $C_{1}$ and $C_{2}$ on both sides of the interface to the growth velocity and gradients of the concentrations. In the equilibrium these two concentrations are just the liquidus and solidus concentrations. When the velocity is finite, these two concentrations deviate from the equilibrium values. We write (see, for example, Ref. [12] and references therein),

$$
\begin{gathered}
\delta \mu_{A} / T=\mathcal{A} J_{A}+\mathcal{B} J_{B}, \\
\delta \mu_{B} / T=\mathcal{B} J_{A}+\mathcal{C} J_{B} .
\end{gathered}
$$

This Onsager matrix should be positive definite: $\mathcal{A}$ and $\mathcal{C}$ must be positive and $\mathcal{B}^{2}<\mathcal{A C}$. According to the conservation of $B$ atoms at the interface we also have [10]

$$
\begin{gathered}
-D_{1} \nabla C_{1}=V C_{1}-J_{B}, \\
-D_{2} \nabla C_{2}=V C_{2}-J_{B}, \\
V=J_{A}+J_{B} .
\end{gathered}
$$

Equation (14) is written for substitutional alloys. For interstitial alloys $V=J_{A}$. For dilute alloys the chemical potential are [13]

$$
\begin{aligned}
& \delta \mu_{A} / T=\left(C_{1}-C_{2}\right)+\left(C_{L}-C_{S}\right), \\
& \delta \mu_{B} / T=\ln \left(C_{2} / C_{1}\right)+\ln \left(C_{S} / C_{L}\right) .
\end{aligned}
$$

Here phase 1 grows at the expense of phase 2. $C_{1}$ and $C_{2}$ are the concentrations of $B$ atoms at the interface and $C_{S}$ and $C_{L}$ are their equilibrium values; $\left(C_{L}-C_{S}\right) \sim\left(T_{M}-T\right) / T$ is proportional to the deviation of the temperature from its equilibrium value for a pure $A$ material. $D_{1}$ and $D_{2}$ are the diffusion coefficients.

According to the mass conservation at the interface for the steady-state $1 \mathrm{D}$ growth, we have $J_{A}=V\left(1-C_{1}\right)$ and $J_{B}=$ $V C_{1}$ because there is no gradient in the growing phase 1. These relations are written for the substitutional alloys. For the interstitial alloys, $J_{A}=V$. However, in the dilute limit there is no difference between these two alloys because $C_{1} \ll 1$ and can be neglected in the expression for $J_{A}$ for the substitutional alloys. Moreover, the global mass conservation requires that $C_{1}=C_{0}$, where $C_{0}$ is the concentration in the original phase 2 far from the interface. Solving the resulting system of equation, we find the transcendental relation between velocity $V$ and the initial concentration $C_{0}$ as follows:

$\ln \left\{\frac{C_{S}}{C_{L}}\left[1+\frac{C_{L}-C_{S}}{C_{0}}-V\left(\mathcal{A} / C_{0}+\mathcal{B}\right)\right]\right\}=V\left[\mathcal{B}+\mathcal{C} C_{0}\right]$.

If the concentration $C_{0}$ is close to $C_{S}$ and the velocity $V$ is small we find, expanding the logarithm up to linear order in $\left(C_{0}-C_{S}\right)$ and $V$,

$$
V=\frac{\left(C_{L}-C_{S}\right)\left(C_{S}-C_{0}\right)}{C_{S}\left[\mathcal{A}+\mathcal{C} C_{L} C_{S}+\mathcal{B}\left(C_{L}+C_{S}\right)\right]}
$$

For the general case of nondilute alloys, this equation reads,

$V=\frac{\left[f_{1}^{\prime \prime}\left(C_{S}\right) / T\right]\left(C_{L}-C_{S}\right)\left(C_{S}-C_{0}\right)}{\mathcal{A}\left(1-C_{L}\right)\left(1-C_{S}\right)+\mathcal{C} C_{L} C_{S}+\mathcal{B}\left[\left(C_{L}+C_{S}\right)-2 C_{L} C_{S}\right]}$,

where $f_{1}^{\prime \prime}(C)$ is the second derivative of the free energy $f_{1}(C)$ of the growing phase 1 with respect to the concentration. From this expression it is clear that in the presence of the cross coefficient $\mathcal{B}$ the sign of $\left(C_{S}-C_{0}\right)$ is not determined by the condition $\mathcal{B}^{2}<\mathcal{A C}$ and also depends on $C_{L}$ and $C_{S}$. Moreover, if the sign in the square brackets of Eq. (18) is negative and $C_{0}>C_{S}$ for small positive velocity $V$ then we find, for $C_{0}=$ $C_{S}$ apart from the solution $V=0$, the second solution with positive $V$. If the expression in the square brackets is negative but small, we can expand the logarithm up to linear order in $\left(C_{0}-C_{S}\right)$ and up to quadratic order in $V$ and find,

$$
\begin{aligned}
\frac{\left(C_{L}-C_{S}\right)\left(C_{S}-C_{0}\right)}{C_{S}}= & V\left[\mathcal{A}+\mathcal{C} C_{L} C_{S}+\mathcal{B}\left(C_{L}+C_{S}\right)\right] \\
& +V^{2}\left[\mathcal{A}+\mathcal{B} C_{S}\right]^{2} /\left(2 C_{L}\right)
\end{aligned}
$$

This expression shows that with increasing $V$ the curve $V=V\left(C_{0}\right)$, first, goes into the two-phase region and then turns back, having another solution with finite velocity at $C_{0}=C_{S}$, and then goes into the one-phase region (see Fig. 1). Eventually, for $C_{0} \rightarrow 0$, the velocity, according to Eq. (17), becomes $V=\left(C_{L}-C_{S}\right) / \mathcal{A} \sim\left(T_{M}-T\right) /(T \mathcal{A})$, as for the solidification of a pure material.

First, we mention the clear analogy between two discussed problems. From the basic equations we see this analogy if we relate $V \rightarrow J_{A}, J_{E} \rightarrow J_{B}, T_{M} S_{1(2)} \rightarrow C_{1(2)}, \delta \mu \rightarrow \delta \mu_{A}$, and $\delta T / T_{M}^{2} \rightarrow \delta \mu_{B} / T$ and apart from some thermodynamical prefactors $\left(\Delta_{T}-1\right) \rightarrow\left(C_{S}-C_{0}\right)$. The case $\delta T=0$ in the pure material problem then corresponds to zero values of $\delta \mu_{B}$. This, in turn, corresponds to a frequently used assumption that the partition coefficient $k=C_{1} / C_{2}$ is equal to its equilibrium value, $k_{0}=C_{S} / C_{L}$. In this case, as in the pure material problem, stationary growth is possible only in the one-phase region of the phase diagram. Actually, it seems that this result is in agreement with the phenomenological description of Refs. [5,8]. 


\section{DISCUSSION AND CONCLUSION: THIN-INTERFACE LIMIT OF PHASE-FIELD MODELS VERSUS THE ONSAGER APPROACH}

We discuss the thin-interface limit using the KR description for the temperature field for a flat interface. The corresponding problem for alloys leads to basically the same results (see, for example, Refs. $[6,8,14])$. Originally it was designed to increase computational power of the method by using larger values of the interface width $W$ and to mimic local equilibrium boundary conditions [3]. Let us have a closer look at this limit from more physical prospectives. In the thin-interface limit of Ref. [3] the temperature distribution $T(x)$ close to the interface is given by $T_{i}(x)=T(0)+G_{i} x$, where $G_{i}$ is the temperature gradient in the $i$-th phase $(i=1,2)$ at $x=0$. At $x=0$ the temperature $T_{1}=T_{2}=T(0)$ and in this description the Kapitza jump is absent, $T_{1}-T_{2}=0$. One should note that the value of $T(0)$ in this linear extrapolation procedure differs from the real value of the smooth temperature field at the middle of the interface obtained by the phase-field simulations. The given linear extrapolation of the temperature field reasonably coincides with direct phase field results only for $H \gg|x| \gg$ $W$, where $W$ is the width of the phase field and $H \gg W$ is some macroscopic length scale. KR derived a kinetic boundary condition that relates the effective temperature $T(0)$ and the growth velocity $V$ by the kinetic coefficient $\mathcal{A}_{\mathrm{KR}}$ : $\left[T_{M}-T(0)\right] L / T_{M}^{2}=\mathcal{A}_{\mathrm{KR}} V$. Using the asymptotic matching procedure, they obtained that the kinetic coefficient has the following structure:

$$
\mathcal{A}_{\mathrm{KR}}=\frac{L^{2}}{T_{M}^{2} c_{p}}\left(\beta_{0}-a \frac{W}{D_{T}}\right),
$$

where $\beta_{0}=1 / V_{0}>0$ is the KR kinetic coefficient in the sharp interface limit $(W \rightarrow 0)$ and $a$ is a positive numerical factor of the order of unity that depends on some tiny details of the specific phase-field model. The second negative term is due to the finite thickness $W$ of the interface and the described linear extrapolation procedure. We also note that in this description the other Onsager coefficients vanish, $\mathcal{B}=\mathcal{C}=0$ in both sharp and and thin-interface limits. KR checked that for the steady-state 1D growth, the analytical prediction, Eq. (8) with the obtained value of $\mathcal{A}_{\mathrm{KR}}$ and $\mathcal{B}=\mathcal{C}=0$, is in good agreement with direct numerical simulations of the phase-field model. However, there is a subtle physical point concerning the interpretation of $\mathcal{A}$, which may become negative with some choice of phase-field-model parameters. As correctly mentioned by KR, this conclusion may appear, at first sight, thermodynamically inconsistent. However, as it has been already mentioned, the temperature $T(0)$ is not a real temperature inside of the interface and deviates strongly from the temperature obtained by phase-field simulation, which is below $T(0)$.

Let us discuss this nontrivial point in more detail. We can imagine an extended interface with the thickness $2 \delta$ with two boundaries located at $x= \pm \delta$. We emphasize that this length scale $\delta$ differs from the phase-field interface width $W$ and is, for the moment, arbitrary, still being much smaller than any relevant macroscopic length scales. We can easily derive the corresponding matrix of Onsager coefficients using the values of $T$ and $\mu$ at the two boundaries of the extended interface as $T_{1}=T(0)-G_{1} \delta$ and $T_{2}=T(0)+G_{2} \delta$ and then $\mu_{1}\left(T_{1}\right)$ and $\mu_{2}\left(T_{2}\right)$. Using Eqs. (5) and (6) we express the temperature gradients $G_{i}$ in terms of $J_{E}$ and $V$, and using Eqs. (3) and (4), we, finally, find the renormalized values of the Onsager coefficients,

$$
\begin{gathered}
\mathcal{A}(\delta)=\mathcal{A}_{\mathrm{KR}}+\mathcal{C}(\delta) T_{M}^{2}\left(S_{1}^{2}+S_{2}^{2}\right) / 2, \\
\mathcal{B}(\delta)=-\mathcal{C}(\delta) T_{M}\left(S_{1}+S_{2}\right) / 2, \\
\mathcal{C}(\delta) T_{M}^{2}=2 \delta / \lambda,
\end{gathered}
$$

where we have assumed that $\lambda_{1}=\lambda_{2}=\lambda$, as in Ref. [3]. A few remarks are in order.

(i) The steady-state result, Eq. (8), is invariant with respect to this renormalization of the Onsager coefficients, i.e., independent of $\delta$. It means that this $\delta$ family of Onsager matrices is in good agreement with numerical simulations of the phase-field model as well as the original KR case, $\delta=0$.

(ii) With the choice $\delta>2 a W$ the matrix of Onsager coefficients becomes positive definite, $\mathcal{A}, \mathcal{C}>0$ and $\mathcal{A C}>\mathcal{B}^{2}$, for arbitrary parameters of the phase-field model. This result has a clear physical meaning. For $\delta \gg W$, we discuss only the range of $|x|$ where the used linear extrapolation of the temperature field is in agreement with the temperature field obtained by the phase field, while for $\delta \ll W$ the temperature at the boundaries strongly deviates from the phase-field description, which is fully thermodynamically consistent by itself. In other words, for $\delta \gg W$, the obtained matrix of kinetic coefficients does describe real physical dissipation in the region $\delta$, while for $\delta \ll W$ this "effective" matrix does not describe the real physical dissipation but still leads to the correct expression for the steady-state growth velocity.

This possible renormalization with $\delta$, much smaller than any macroscopic length scale $H$, is not specific only to the phase-field models and represents a small effect of the order of $\delta / H \ll 1$. It has the same structure as the "negative" phase-field effects $W / H$. The ideology of any macroscopic description relies on this small parameter as an expansion parameter of the theory. These corrections should be irrelevant in the general case of the diffusional transformation where the bulk dissipation plays the major role (for example, in the case of dendritic growth at small undercooling). We have seen, however, that in the specific problem of steady-state 1D front propagation, this small term (proportional to $W$ ) is responsible for the sign of the slope in the phase-field model description. This happens because the bulk dissipation (being still much larger than the interfacial dissipation) just bring us to the vicinity of point $\Delta_{T}=1$ and does not contribute to the slope. In this case, the growth velocity is entirely controlled by the interface kinetics. We note that the interpretation of the nontrivial behavior in the vicinity of $\Delta=1$ due to sufficiently negative values of the phenomenological cross coefficient $\mathcal{B}$ does not assume any specific model of the interface. At the same time, the explanation suggested by the phase-field modeling explicitly takes inhomogeneities of the temperature and concentration fields, on the scale of finite interface thickness, into account.

In other words, there is no doubt about thermodynamic consistency of the phase-field model for arbitrary values of the parameter $V_{0} W / D_{T}$ apart from the obvious restrictions, 
$V_{0}>0$ and $D_{T}>0$. However, the interpretation of the thininterface limit and its relation to the matrix of dissipative Onsager coefficients should be taken with care. We illuminate this warning by the following additional example. Let us assume that, initially, the two-phase system is at some temperature $T$ slightly below the melting temperature $T_{M}$. This system evolves toward equilibrium with a solidification velocity $V$ that decays as $V \sim t^{-1 / 2}$ at large time $t$. This behavior would be observed in direct phase-field simulations for arbitrary parameters of the model and independent of the sign of the effective kinetic coefficient, Eq. (21). A slightly different, but close in spirit, nonstationary evolution has been discussed in Ref. [3] confirming this behavior. However, if one solved this problem not by a direct phasefield simulation but by solving the free boundary problem with effective boundary conditions described by the the matrix of kinetic coefficients, $\mathcal{A}=\mathcal{A}_{\mathrm{KR}}$ and $B=C=0$ (the thin-interface limit of Ref. [3]), the result would differ markedly if $\mathcal{A}_{\mathrm{KR}}<0$. The system would melt, instead of being solidified, exhibiting strong instabilities and would never reach the described physical attractor. On the other hand, if one solved the same problem using the renormalized positivedefinite matrix of Onsager coefficients, Eqs. (22)-(24), the result would be basically the same as in direct phase-field simulations and physically relevant. Therefore, we conclude that the interpretation of the thin-interface limit of Ref. [3] as the correspondence between the phase-field description and the classical macroscopic approach is incorrect for the wide class of nonstationary problems if $\mathcal{A}_{\mathrm{KR}}<0$. However, the renormalized positive-definite matrix of Onsager coefficients leads to such a correspondence in the macroscopic limit for arbitrary $\mathcal{A}_{\mathrm{KR}}$.

Additionally, we address one more point. The phase-field model of Ref. [3] contains fewer independent parameters to describe the kinetic properties of the interface (only $\mathcal{A}$ or $\beta_{0}$ ) than is allowed by the general phenomenology $(\mathcal{A}, \mathcal{B}, \mathcal{C})$. While an independent parameter $\mathcal{C}$ can be introduced in a slightly modified version of the phase-field model, the introduction of the independent cross coefficient $\mathcal{B}$ is a serious problem. As pointed out in Ref. [15], according to Curie's principle
[16], there can be no kinetic coupling between the scalar nonconserved phase-field order parameter $\phi$ and vectorial diffusional fluxes of the conserved quantities energy and/or concentration. Thus, one should not expect an independent cross coefficient $\mathcal{B}$ to appear in the effective boundary conditions, Eqs. (3), (4), (10), and (11). However, in the general case of the phenomenological macroscopic description, we do not doubt the existence of such a kinetic coupling at the interface between the normal growth velocity and normal diffusional fluxes through the interface. It is conceivable that this coupling can be introduced in modified versions of the phase-field model where $\nabla \phi /|\nabla \phi|$, the unit vector normal to the interface, can be used to produce the corresponding vectorial quantities. This issue may also be relevant to the antitrapping current introduced in some nonvariational versions of the phase-field model $[14,17]$ for different purposes. The antitrapping current introduces a new kinetic coefficient and uses the unit vector normal to the interface. To use this idea for the description of the cross effect of the interface kinetics in phase-field models, one should carefully consider the necessary symmetry that is obligatory for this cross effect. A more detailed discussion of this question is far beyond the scope of this paper.

Finally, we stress that the interface kinetics and its proper description is very important for many other interesting phenomena observed in solidification of binary alloys and also in solid-solid transformations. A very nice and deep discussion of solute trapping and its relation to cross terms of the Onsager matrix is given in Ref. [12]. Another example is the oscillation of the solidification front in a binary alloy which is growing in the setup of directional solidification. In many metallic alloys this leads to the formation of banded structures [18]. For very recent achievements in this direction and related phenomena, see Ref. [19] and references therein.

\section{ACKNOWLEDGMENTS}

We thank M. Plapp for useful discussions. We acknowledge the support of the Deutsche Forschungs-gemeinschaft under Project No. SFB 917.
[1] N. Provatas and K. Elder, Phase-Field Methods in Materials Science and Engineering (Wiley-VCH Verlag $\mathrm{GmbH}$, Weinheim, Germany, 2010).

[2] Y. Saito, Statistical Physics of Crystal Growth (World Scientific, Singapore, 1996).

[3] A. Karma and W. J. Rappel, Phys. Rev. E 53, R3017 (1996); 57, 4323 (1998).

[4] H. Löwen, J. Bechhoefer, and L. S. Tuckerman, Phys. Rev. A 45, 2399 (1992).

[5] M. J. Aziz and W. J. Boettinger, Acta Metall. Mater. 42, 527 (1994).

[6] K. R. Elder, M. Grant, N. Provatas, and J. M. Kosterlitz, Phys. Rev. E 64, 021604 (2001).

[7] A. Umantsev, J. Chem. Phys. 96, 605 (1991).

[8] A. L. Korzhenevskii, R. Bausch, and R. Schmitz, Phys. Rev. E 83, 041609 (2011).

[9] G. Boussinot and D. E. Temkin (unpublished).
[10] D. E. Temkin, J. Cryst. Growth 52, 299 (1981).

[11] S. Balibar, H. Alles, and A. Ya. Parshin, Rev. Mod. 77, 317 (2005), (see p. 347).

[12] B. Caroli, C. Caroli, and B. Roulet, Acta Metall. 34, 1867 (1986).

[13] L. D. Landau and E. M. Lifshitz, Statistical Physics (Pergamon Press, Oxford, 1963).

[14] A. Karma, Phys. Rev. Lett. 87, 115701 (2001).

[15] Z. Bi and R. F. Sekerka, Physica A 261, 95 (1998).

[16] S. R. De Groot and P. Mazur, Non-Equilibrium Thermodynamics (North-Holland, Amsterdam, 1962).

[17] B. Echebarria, R. Folch, A. Karma, and M. Plapp, Phys. Rev. E 70, 061604 (2004).

[18] M. Carrard, M. Gremaud, M. Zimmermann, and W. Kurz, Acta Metall. Mater. 40, 983 (1992).

[19] A. L. Korzhenevskii, R. Bausch, and R. Schmitz, Phys. Rev. Lett. 108, 046101 (2012). 